\title{
BMJ Open Study protocol for a prospective, non-controlled, multicentre clinical study to evaluate the diagnostic accuracy of a stepwise two-photon excited melanin fluorescence in pigmented lesions suspicious for melanoma (FLIMMA study)
}

\author{
Christine Fink, ${ }^{1}$ Maja Hofmann, ${ }^{2}$ Agata Jagoda, ${ }^{2}$ Iris Spaenkuch, ${ }^{3}$ \\ Andrea Forschner, ${ }^{3}$ Ioanna Tampouri, ${ }^{3}$ Diana Lomberg, ${ }^{3}$ Dieter Leupold, ${ }^{4}$ \\ Claus Garbe, ${ }^{3}$ Holger A Haenssle ${ }^{1}$
}

To cite: Fink C, Hofmann M, Jagoda A, et al. Study protocol for a prospective, non-controlled, multicentre clinical study to evaluate the diagnostic accuracy of a stepwise two-photon excited melanin fluorescence in pigmented lesions suspicious for melanoma (FLIMMA study). BMJ Open 2016;6: e012730. doi:10.1136/ bmjopen-2016-012730

- Prepublication history for this paper is available online. To view these files please visit the journal online (http://dx.doi.org/10.1136/ bmjopen-2016-012730).

Received 19 May 2016 Revised 21 September 2016 Accepted 30 September 2016

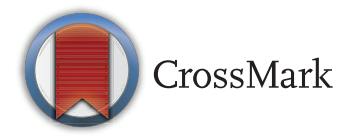

For numbered affiliations see end of article.

Correspondence to Holger Haenssle; Holger.Haenssle@med. uni-heidelberg.de

\section{ABSTRACT}

Introduction: Non-invasive, nanosecond, stepwise two-photon laser excitation of skin tissue was shown to induce melanin fluorescence spectra that allow for the differentiation of melanocytic nevi from cutaneous melanoma.

Methods and analysis: This prospective, non-controlled, multicentre clinical study is performed to evaluate the diagnostic performance of the stepwise two-photon excited melanin fluorescence in the detection of cutaneous melanoma. The comparator will be the histopathological diagnosis. A total of 620 pigmented skin lesions suspicious for melanoma and intended for excision will be enrolled.

Ethics and dissemination: Ethics approval was provided by the local ethics committees of the medical faculties of the University of Tuebingen, Heidelberg and Berlin.

Study registration: The FLIMMA study NCT02425475.

\section{INTRODUCTION}

\section{Background}

Melanoma is a malignant tumour, which develops from the pigment producing melanocytes by neoplastic transformation. Malignant melanoma is identified as one form of cancer of increasing incidence, which is now among the 10 most frequent human malignancies. In Europe, the regional incidence rates reside between 10 and 25 per 100000 per year and increase, especially in elderly people. ${ }^{1-6}$ After diagnosis of a malignant melanoma, the
Strengths and limitations of this study

- For the first time, the diagnostic performance of non-invasive, nanosecond, stepwise two-photon laser excitation in the detection of melanoma wil be evaluated.

- The University of Tuebingen acts as the sponsor of the study, thus reducing the influence of commercial interests and bias.

- The FLIMMA study is designed as a prospective multicentre observational study. Histopathologists are blinded to the results of the test device and a central review board of histopathologists will review all false-negative results of the test device. While randomised controlled trials generate the most reliable evidence, the protocol described here is a necessary preliminary step in this challenging area of research and was closely adapted from Food and Drug Administration-approved protocols in the field of medicinal products for the diagnosis of melanoma.

prognosis depends strongly on the thickness of the tumour and lymphatic or haematogenous metastases. ${ }^{7}$ Once melanomas cause metastases, the prognosis of survival worsens dramatically, with a 5 -year survival of patients with stage IV disease between $6.7 \%$ and $18.8 \% .^{8}$ This underlines the urgent need to diagnose melanoma as early as possible. The diagnostic accuracy for the clinical melanoma diagnosis does not exceed $75 \%$ and may be increased to up to $90 \%$ by the use of dermoscopy in the hands of experts; however, $\sim 10 \%$ of melanomas will be missed despite all these aforementioned diagnostic efforts. The term 'featureless 
melanoma' has been coined for this phenomenon. ${ }^{10}$ Particularly in these difficult to diagnose and 'featureless' melanomas, additional strategies for melanoma diagnosis would be extremely helpful.

\section{Two-step photon excitation of melanin fluorescence}

Autofluorescence spectra of human skin tissue are usually excited by one-photon absorption in the ultraviolet-A region. ${ }^{11}$ However, by this form of excitation, the ultra-weak fluorescence of melanin is undetectably hidden by the emission from the main endogenous fluorophores NAD(P)H and flavins. ${ }^{12} 13$ A more specific excitation of the melanin fluorescence may be useful for gaining information about the potential malignancy of pigmented skin lesions. A first step to overcome this lack of specific melanin fluorescence was the application of more targeted fluorescence excitation technique based on two photon absorption from an $800 \mathrm{~nm}$-femtosecond laser. All endogenous skin tissue fluorophores except melanin do not absorb at $800 \mathrm{~nm}$; nevertheless, on irradiation with $800 \mathrm{~nm}$-laser pulses, they may show their well-known fluorescence in the spectrum of visible light. This is caused by a special non-linear optical effect called simultaneous two photon absorption. The intensity of the excited fluorescence is comparably weak. In contrast, melanin shows absorption on irradiation with $800 \mathrm{~nm}$-laser pulses and absorbs two photons in a stepwise process via an intermediate excited electronic state. ${ }^{12}$ At physiologically acceptable laser intensities, the latter mechanism is much more effective. ${ }^{14}$ By this procedure, the main autofluorescence of skin will be partly suppressed and the melanin fluorescence becomes measurable. Investigations in a variety of pigmented skin lesions gave first hints on the differences between the fluorescence from common nevi as compared to malignant melanoma. ${ }^{15}$ Also, in other melanin-containing fluorophore compositions, this fluorescence discrimination in favour of melanin can be observed, for example, in the choroidea and the retinal pigment epithelium of the eye. ${ }^{16}$ A further essential improvement in measuring the melanin fluorescence from skin tissue as selectively as possible could be achieved only recently by using nanosecond pulses instead of femtosecond pulses. $^{17}$

\section{The dermatofluoroscope Magnosco DFC 1 for in vivo diagnostics of melanoma}

The investigational device in this study is the dermatofluoroscope Magnosco DFG 1 by Magnosco $\mathrm{GmbH}$, Berlin, Germany, with a two-photon excitation with $800 \mathrm{~nm} /$ nanosecond pulses from a dye laser, equipped with a spectrometer and a sensitive photon detector. It is designed for use by dermatologists and trained medical personnel and should be applied to patients with skin types I, II, III and IV, who show atypical melanocytic lesions. For the investigation with the Magnosco DFC 1, the patient has to be at physical rest. The overview CCD camera is applied to take a macroscopic image for documentation reasons. After cleaning and shaving the location of interest, the lesion is covered with a specific cover shield with mask. The latter helps to place the scanning head onto the intact skin. After fixing it by an adhesive pad, a dermoscopic image of the lesion is taken. The spectral data are gathered automatically, while the lesion is raster-scanned. The results of the data analysis are presented on the computer screen: (1). Dermoscopic image overlaid with the scanning raster: fluorescence spectra indicating malignancy are visualised as red spots. (2). A score given on a green/red bar indicates the result of data analysis: (a) presence of malignant melanoma, (b) no indication of malignant melanoma, (c) no valid result. The analysis of the spectral data in conjunction with images and patient master data are documented as one file in the dermatofluoroscope Magnosco DFC 1 database. All files can be transferred with the customised USB stick or printed for patient information.

\section{Preliminary data with the Magnosco DFC 1 device}

The preclinical data available for assessing the suitability of the dermatofluoroscope Magnosco DFC 1 for melanoma diagnostics are based on ex vivo and histological specimen examination. The specimens examined so far were freshly excised pigmented nevoid lesions and their corresponding paraffin-embedded histological samples. In 167 freshly excised tissue specimens from clinically suspicious pigmented lesions (suspected malignant melanoma/dysplastic nevi), the diagnosis was first made based on the new fluorescence-spectroscopic diagnostic method before the histopathological diagnosis was available. In relation to the histopathological diagnosis as the current gold standard of melanoma diagnostics, the new diagnostic method showed a sensitivity of melanoma detection of $93.5 \%$, a specificity of $80.0 \%$ and a diagnostic accuracy of $82.6 \%$ on freshly excised pigmented lesions. $^{18}$ In a study on 125 paraffin embedded specimens of melanocytic melanomas $(n=60)$ and melanocytic nevi $(n=65)$, a sensitivity of melanoma detection of $82.5 \%$ and a specificity of $72.5 \%$ were detected. ${ }^{19}$

\section{DESIGN/METHODS \\ Study design}

The FLIMMA study is designed as a prospective, noncontrolled, multicentre clinical study in patients with suspected malignant melanoma.

\section{Objectives}

The primary objective of this study is to determine the sensitivity and specificity of the algorithm for the fluorescence diagnostics of melanoma. The comparator and gold standard for the diagnosis will be the histopathological diagnosis of the pigmented lesions. Secondary objectives are to collect data for training and optimisation of the diagnostic algorithm, and to assess the safety of the device and the incidence of adverse events. 


\section{End points}

The primary end point is to determine the sensitivity and specificity of this fully automated, non-invasive, in vivo method. Secondary end points include the assessment of the safety of the device as well as the collection of data for training and optimisation of the computerised diagnostic algorithm.

\section{Recruitment and status of the study}

The date of first enrolment was 17 September 2014. The recruitment of patients is in progress. The estimated total time frame for recruitment of 620 patients is 20 months. The total duration of the study is expected to be 26 months, including analysis.

\section{Study population}

A total of 620 patients, who show pigmented lesions with suspicion of dysplastic nevus or melanoma and in whom an excision is indicated, will be recruited.

\section{Criteria for inclusion/exclusion}

Patients having pigmented lesions with a suspicion of dysplastic nevus or melanoma, in whom an excision is indicated in order to exclude or diagnose malignant melanoma, who are $\geq 18$ years of age, and who have given written informed consent will be eligible. Patients with skin types V and VI according to Fitzpatrick's scale; patients where there is a risk that the scanning head is detached because the patient cannot be placed at rest, patients who cannot understand the patient information and who cannot provide informed consent, patients with deep dermal lesions $\geq 5 \mathrm{~mm}$ beneath the stratum corneum, clinically or dermoscopically obviously nonmelanocytic lesions, periungual and subungual lesions, mucosal lesions, lesions with trauma, erosion, excoriation or ulceration on more than $50 \%$ of the lesion area, tattooed lesions, patients suffering from albinism, pregnant or breastfeeding women, lesions with dominant $(>50 \%)$ regression and lesions which are not suitable to fix the scanning head will be excluded from the FLIMMA study.

\section{Methods}

A total of 620 pigmented skin lesions intended for excision to either confirm or rule out melanoma will be enrolled after written informed consent at the participating centres. Three centres participated within Germany: University Hospital of Tuebingen as the lead centre, University Hospital of Heidelberg and Charité Berlin. Clinical and dermoscopic images will be recorded for all cases. Then, as a second diagnostic procedure, fluorescence diagnostics based on the two-photon excitation from a dye-laser will be performed. The classification as non-melanoma or malignant melanoma by the medical dermatofluoroscope Magnosco DFC 1 will be documented prior to the excision. The in vivo melanin fluorescence assessment will be performed no longer than 14 days prior to excision. Histopathologists on the study sites will be blinded to the diagnoses attained by the analyses of fluorescence spectra. Moreover, all falsenegative cases with a disagreement in the diagnosis by the test method and the histopathological examination on site will be submitted to a blinded central pathology review board. The histopathological diagnosis will serve as a gold standard for subsequent evaluations of the diagnostic accuracy. The FLIMMA study was registered at ClinicalTrials.gov (Identifier: NCT02425475).

\section{Statistical considerations}

In this study, 560 evaluable lesions will be recruited, including 80 evaluable melanomas. In order to compensate for any dropouts, a total of 620 specimens are examined. It is assumed that the true sensitivity is in the order of $90 \%$, and the true specificity is in the order of $35 \%$ based on the available preclinical data described in more detail in the introduction section. With 70 evaluable melanoma specimens and an observed sensitivity of $90 \%$, the two-sided $95 \%$ CI is 0.80 to 0.96 . The specificity is assumed to be in the order of $35 \%$, which is evaluated in 420 specimens, according to negative gold standard specimen results in a CI of 0.30 to 0.40 . This accuracy is considered sufficient for the method to be evaluated. The study data and the cohort under evaluation will be analysed by means of descriptive statistics. Furthermore, the main end point of the present study is the diagnostic accuracy. The sensitivity and specificity of the diagnostic method of fluorescence-based pigment analysis will be determined. The comparator and goal standard for this analysis is the histopathological diagnosis.

\section{ETHICS AND DISSEMINATION \\ Declarations and ethic aspects}

The study is conducted in accordance with the Declaration of Helsinki principles (2013) ${ }^{20}$ requirements and guidance provided in ISO 14155 (2012) ${ }^{21}$ and applicable local government regulations and Independent Ethics Committee policies and procedures. In the context of the approved standard operating procedures which are based on ICH-GCP guidelines (E6) and the German implementation of Good clinical practice (GCP) for the clinical work, the patients will be informed orally and in written form about the aim, character and consequences of the procedure. Before initiation of the study, the protocol, the patient information sheet and the consent form were presented to the independent ethics committee. The names of patients and all confidential data are subject to professional discretion and the 'Bundesdatenschutzgesetz (BDSG)'. Processing of medical data will only take place in pseudonymous form. In case of withdrawal from the study, the data that have already been collected will be destroyed. Each participant will be informed that participation in the study is voluntary and that he/she may withdraw from the study at any time and that withdrawal of consent will not affect his/ her subsequent medical assistance and treatment. The investigator will explain to each participant the nature of 
the study, its purpose, the procedure involved, the expected duration, the potential risks and benefits and any discomfort it may entail. Additionally, all participants for the study will be provided a participant information sheet and a consent form describing the study and providing sufficient information for participants to make an informed decision about their participation in the study. The formal consent of a participant, using the approved consent form, must be obtained before the participant is submitted to any study procedure. The participant should read and consider the statement before signing and dating the informed consent form, and be given a copy of the signed document. The consent form must also be signed and dated by the investigator and it will be retained as part of the study records. All records relating to this study are stored in an external archive and must be retained for at least 10 years after completion of the research.

\section{Risk-benefit relationship}

The decision for excision is based on the clinical and dermoscopic diagnosis of the pigmented lesion, and will not be biased by the diagnosis of the device under investigation (dermatofluoroscope Magnosco DFC 1). The melanin fluorescence measurements of the FLIMMA study will not influence the clinical procedures. Therefore, there is no risk for the patient that his participation may deteriorate the management rate of his pigmented lesions. The information of the patient and the measurement procedure itself will generally take $<15 \mathrm{~min}$. In case of a false-negative diagnosis by the dermatofluoroscope Magnosco DFC, a second independent histopathological review will be performed. This will be in favour of a higher diagnostic accuracy and may be beneficial for the patient.

\section{SAFETY}

This is a non-invasive diagnostic procedure based on a low-intensity visible light exposure, which has no capacity to injure tissues. Therefore, no adverse reactions related to the optical procedures are expected. All adverse events (AEs) will be recorded and documented. Serious AE will be reported in accordance with the Medizinprodukte-Sicherheitsplanverordnung (MPSV) ordinance.

\section{Author affiliations \\ ${ }^{1}$ Department of Dermatology, University of Heidelberg, Germany \\ ${ }^{2}$ Department of Dermatology, Charité Berlin, Berlin, Germany \\ ${ }^{3}$ Department of Dermatology, University of Tübingen, Tübingen, Germany \\ ${ }^{4}$ Magnosco GmbH, Deuben, Germany}

Contributors $\mathrm{HAH}, \mathrm{CG}$ and $\mathrm{MH}$ participated in the development and implementation of the study (sample size calculations, writing of the protocol, submission to ethics committee, data management). IS, DL and CG performed the data handling and statistical analysis. CF, AF, IT, AJ and DL helped to draft and review the paper. All authors read and approved the final manuscript.

Funding The University of Tuebingen acted as the sponsor of the study. This research received no grant from a commercial sector.
Competing interests None declared.

Ethics approval Ethics approval was provided by the local ethics committees of the medical faculties of the University of Tuebingen, Heidelberg and Berlin.

Provenance and peer review Not commissioned; externally peer reviewed.

Open Access This is an Open Access article distributed in accordance with the Creative Commons Attribution Non Commercial (CC BY-NC 4.0) license, which permits others to distribute, remix, adapt, build upon this work noncommercially, and license their derivative works on different terms, provided the original work is properly cited and the use is non-commercial. See: http:// creativecommons.org/licenses/by-nc/4.0/

\section{REFERENCES}

1. Stang A, Stang K, Stegmaier C, et al. Skin melanoma in Saarland: incidence, survival and mortality 1970-1996. Eur J Cancer Prev 2001;10:407-15.

2. Garbe C, Leiter U. Melanoma epidemiology and trends. Clin Dermatol 2009;27:3-9.

3. Lasithiotakis KG, Leiter U, Gorkievicz R, et al. The incidence and mortality of cutaneous melanoma in Southern Germany: trends by anatomic site and pathologic characteristics, 1976 to 2003. Cancer 2006;107:1331-9.

4. Lasithiotakis KG, Petrakis IE, Garbe C. Cutaneous melanoma in the elderly: epidemiology, prognosis and treatment. Melanoma Res 2010;20:163-70.

5. Leiter U, Garbe C. Epidemiology of melanoma and nonmelanoma skin cancer-the role of sunlight. Adv Exp Med Biol 2008;624:89-103.

6. Bosetti C, La Vecchia C, Naldi L, et al. Mortality from cutaneous malignant melanoma in Europe. Melanoma Res 2004;14:301-9.

7. Tsao H, Atkins MB, Sober AJ. Management of cutaneous melanoma. N Engl J Med 2004;351:998-1012.

8. Balch CM, Soong SJ, Gershenwald JE, et al. Prognostic factors analysis of 17,600 melanoma patients: validation of the American Joint Committee on Cancer melanoma staging system. J Clin Oncol 2001;19:3622-34.

9. Weide B, Elsasser M, Buttner $P$, et al. Serum markers lactate dehydrogenase and $\mathrm{S} 100 \mathrm{~B}$ predict independently disease outcome in melanoma patients with distant metastasis. $\mathrm{Br} \mathrm{J}$ Cancer 2012;107:422-8.

10. Menzies SW, Ingvar C, Crotty KA, et al. Frequency and morphologic characteristics of invasive melanomas lacking specific surface microscopic features. Arch Dermatol 1996;132:1178-82.

11. Richards-Kortum R, Sevick-Muraca E. Quantitative optical spectroscopy for tissue diagnosis. Annu Rev Phys Chem 1996;47:555-606.

12. Teuchner K, Freyer W, Leupold D, et al. Femtosecond two-photon excited fluorescence of melanin. Photochem Photobiol 1999;70:146-51.

13. Zeng $\mathrm{H}$, MacAulay $\mathrm{C}$, McLean DI, et al. Spectroscopic and microscopic characteristics of human skin autofluorescence emission. Photochem Photobiol 1995;61:639-45.

14. Teuchner K, Ehlert J, Freyer W, et al. Fluorescence studies of melanin by stepwise two-photon femtosecond laser excitation. J Fluoresc 2000;20:275-81.

15. Hoffmann K, Stucker M, Altmeyer P, et al. Selective femtosecond pulse-excitation of melanin fluorescence in tissue. J Invest Dermatol 2001;116:629-30.

16. Schneider M, Teuchner K, Leupold D. [Two-photon fluorescence of ocular melanomas. Studies on a new diagnostic method] Ophthalmologe 2005;102:703-7.

17. Eichhorn R, Wessler G, Scholz M, et al. Early diagnosis of melanotic melanoma based on laser- induced melanin fluorescence. J Biomed Opt 2009;14:034033.

18. Leupold D, Scholz M, Stankovic G, et al. The stepwise two-photon excited melanin fluorescence is a unique diagnostic tool for the detection of malignant transformation in melanocytes. Pigment Cell Melanoma Res 2011;24: 438-45.

19. Freudenberger S. [Informationsgehalt der Melanin-dominierten Fluoreszenz pigmentierter kutaner Läsionen in Paraffin]. [Dissertation]. Universitätsklinik Tübingen, 2014.

20. World Medical Association. World Medical Association Declaration of Helsinki: ethical principles for medical research involving human subjects. JAMA 2013;310:2191-4.

21. Clinical investigation of medical devices for human subjects-good clinical practice (ISO 14155:2011 + Cor. 1:2011); German version EN ISO 14155:2011 + AC:2011. 